\title{
29 Environmental systems innovation in ancient India with Factor X components as revealed in old Tamil Manuscripts
}

\author{
Kannan Narayanan
}

\section{Introduction}

The post-industrial revolution has changed our paths, personalities, lifestyle and perspectives on a global scale. As a result, we have forgotten our roots and our civilisational continuity. There is a quick search for solutions to a world in disorder. Our present-day market economy has ultimately changed humans into gregarious consumers who are tilting the balance of nature, exhausting resources by exceeding its replenishing capacity. Our habitat has been changed, life forms (flora and fauna) have been exterminated, food chain has been poisoned, and the world has been polluted with anthropogenic hazardous materials (El-Haggar 2007). Part of the problem is that the global system is so massive that we could not comprehend the cause but the aftermath can be seen and felt globally. Climate crisis is an excellent example which has altered seasonal monsoons everywhere, affecting our food production leading to poverty (O'Connor 2019). Monocultural practice in agriculture has placed humanity in peril. If an epidemic on the few principle crops that sustain us occurs, it will be a disaster. Biodiversity has been reduced through landscaping and cash crop agriculture. What we are witnessing on a global scale, could be just the tip of the iceberg. The ramifications of such a catastrophe have not yet been completely understood.

Though planet earth functions as an enclosed ecosystem in unison with its components, people who inhabit it are not in harmony with nature, but divisive in thinking and antagonistic to each other and nature. Their politics, economy and local governance are disruptive. It was only in the "60s that an interdisciplinary field called Ecology was introduced in university education, to understand how nature works. Realisation soon followed that our ecosystem was in trouble and human activities were the cause for it. Rachel Carson's Silent Spring broke the 'God's in His heaven, all is right with the world' lull. (Mann 2018). This was largely due to our misconstruction that (a) earth's resources are limitless and renewable, (b) our actions in polluting the waters, air and land are local and hence will not have far-reaching effects, (c) the belief that man is special and everything else 'out there' is of no concern to him. These wrong beliefs led to indifference and apathy, which in turn are the main reasons for his present existential crisis. 
The current global crisis is new to humanity. However, it has seen civilisational declines due to environmental disaster or mismanagement of local resources. For example, the Mayan civilisation collapsed due to overpopulation and overuse of the land, endemic warfare and drought. Some researchers point out that the loss of topsoil was a principle factor in the collapse of Mayan agriculture which, led to famine and the ultimate destruction (History.com 2019). 'The medieval Khmer were confronted with a period of climatic instability that they had no experience of, and which fully changed the rules of the game that they had been playing for hundreds of years,' said Dan Penny, an expert in environmental history who has studied the Angkor civilisation for many years (Lovgren 2019). A similar scale of challenge is now confronting contemporary communities, as the climate begins to change.

If Factor $\mathrm{X}$ stands for savings in natural resources and resource efficiency improvements, then one finds examples in ancient Tamil texts as explained below. It is indeed comforting to realise that humanity has thought about it earlier. The nexus perspective emphasises the inter-relatedness and interdependencies of these environmental resources and their transitions and fluxes across spatial scales and between compartments. Surprisingly, these principles were well understood and addressed in ancient times. United Nations has produced a set of universal goals that meet the urgent environmental, political and economic challenges facing our world. Centuries ago, the wise people of southern India had addressed these Sustainable Development Goals (SDGs 2019). Observing nature's connectivity, they came up with concepts of circular economy as well. Thus, this

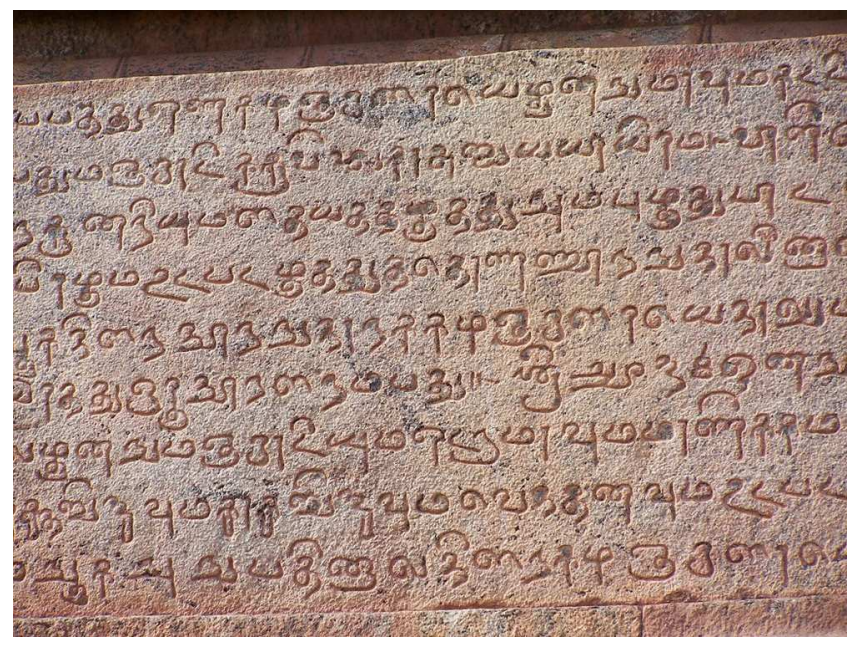

Figure 29.1 Stone inscriptions in Tamil (11 CE).

Source: Wikipedia 
article attempts to remind scholars of an ancient past voicing the same concerns of the current world (Jackson 2017).

\section{Methodology}

The ancient wisdom of Tamil culture, an integral part of Indian civilisation, was understood relatively recently, after the arrival of Europeans to India along with the introduction of modern press (Encyclopaedia Britannica 2017). Printing technology encouraged Tamil scholars to transfer ancient knowledge from an older media to a newer one. There was a hunt for palm leaf manuscripts that carried ancient wisdom to be transformed into print media. Publication, reproduction and distribution of these were factors that encouraged Tamil scholars in early 20th century to read, undertake discourse analysis and publish ancient knowledge to an emerging modern world. Unfortunately, those who are familiar with classical Tamil are getting scarce these days. Additionally, there are not that many among the scholars who can understand those ancient scripts and interpret them in Factor $\mathrm{X}$ and Nexus perspective. The author of this manuscript whose speciality is environmental sciences and whose mother tongue is Tamil however, manages to link the ancient wisdom and modern nexus perspective. Fortunately, most of the ancient Tamil texts are available in the web which has helped the author to collect those poems from cyberspace. Interactive social media have enabled the author to interact with other Tamil scholars to verify the meaning and perspective of those ancient Tamil voices. While Thirukkural, a well-known treatise in Tamil, reflecting the ancient wisdom of the land had been translated into English by early European scholars such as Rev. G.U. Pope (Tirukkural 1886), most of the other poems in this article have no published translations.

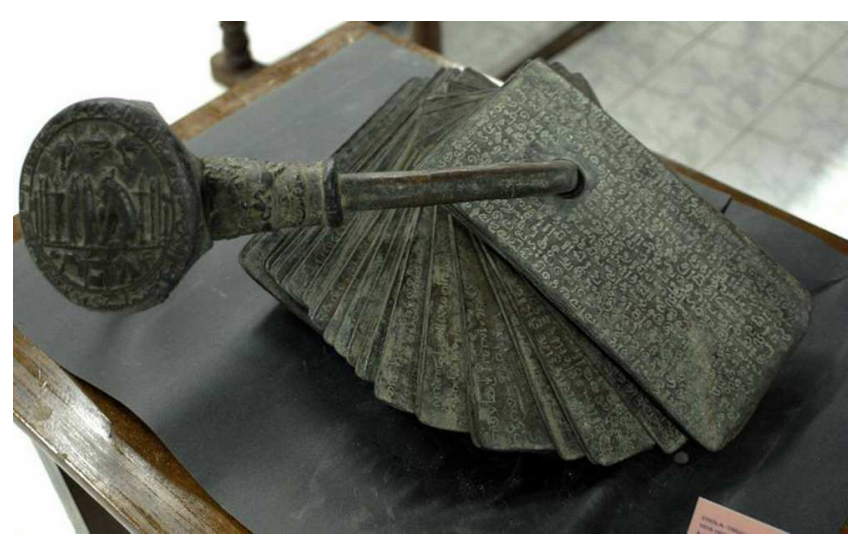

Figure 29.2 The Tiruvalangadu copper plates of Rajendra Chola (reign: 1018-1048), which records his gift of the Palaiyanur village to the Tiruvalangadu Siva temple.

Source: photo by R. Shivaji Rao. 


\title{
Results and discussion
}

Human conflicts have severed man's links with ancient wisdom and a lifestyle that was originally eco-friendly and eco-centric. Browsing through the ancient manuscripts of an older civilisation in the southern part of India where people speak one of the oldest languages of the world, namely, Tamil, one gets an idea of how those people discovered the planetary boundaries, ecological limitations and attempted to live a lifestyle that was pro-environmental. Nature was at the centre of their life, whether in love, business, agriculture, tribal governance or travel. Their correct understanding of the natural system in which they lived, made them an integral part of a unifying universe. This is completely different from a Judeo-Christian perspective that placed man at the centre of the universe. This naturally led to the assumption that nature is for them to exploit. An eventual development was a growing apathy towards nature and a complete indifference towards it to treat the earth as a dust bin for his trash (Denig 1985).

However, discussions in recent years on a global level, about sustainable use of natural resources and the implementation of resource efficiency improvements, have picked up considerable speed and shaped the environmental debate. In 2017, there was a decision to establish a G20 Resource Efficiency Dialogue to make efficient and sustainable use of natural resources, a core element of future.

While these efforts look modern and timely, it may come as a surprise to know that sustainability principles were elucidated by ancient Tamil scholars, particularly in this case as an advice to the king who ruled their land (Pisiranthaiyar 2019). A poem, written roughly about 400 years before the Christian era, speaks eloquently about sustainability and renewability. This poem was quoted recently (2019 Union Budget) in the Indian parliament by Nirmala Seetharaman, the Finance minister of Union of India. The poem reads:

\author{
காய்நெல்அறுத்துக்கவளங்கொளினே, \\ மாநிறைவுஇல்லதும்பன்னாட்குஆகும், \\ நூறுசெறுஆயினும்தமித்துப்புக்குஉணினே, \\ வாய்புகுவதனினும்கால்பெரிதுகெடுக்கும், \\ அறிவுடைவேந்தன்நெறிஅறிந்துகொளினே, \\ கோடியாத்துநாடுபொிதுநந்தும், \\ மெல்லியன்கிழவன்ஆகிவைகலும் \\ வரிசைஅறியாக்கல்லென்சுற்றமொடு \\ பரிவுதபஎடுக்கும்பிண்டம்நச்சின், \\ யானைபுக்கபுலம்போலத், \\ தானும்உண்ணான், உலகமும்கெடுமே
}


The author Pisiranthaiyar explains the principles of sustainability using paddy field and elephant as an example. If paddy cultivation is allowed to fruition, the harvested rice can feed an elephant for months. On the contrary, if one allows the elephant to feed on its own, most of the yield will be trampled and wasted. A king should know the principles of sustainability and govern his land accordingly. Instead, if he is driven by greed and by wrong advisers, he will not only destroy it but will cause suffering on a large-scale. The poet talks about natural resources and resource-efficiency improvements through good practice or best practice in line with the modern understanding of Factor X.

In another incident, a piece of advice was given to the king by a scholar named Kudapulaviyanar (2019) which is as follows:

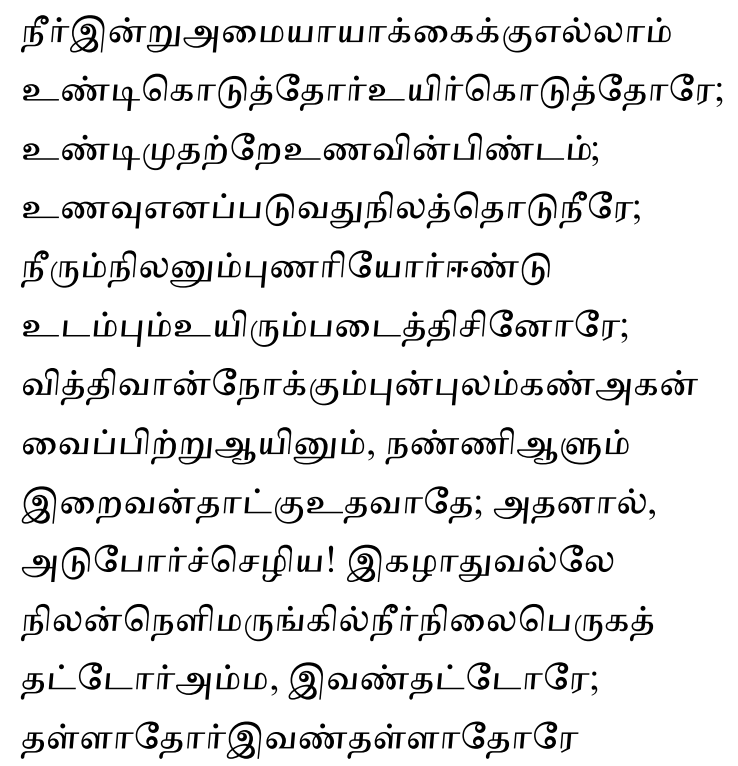

This author had a clear understanding of planet earth and its functions. He explains the intrinsic bond of water to planet earth and its intricate link to life. He states that food is a combination of soil and water. Watering the land is giving life to it. Therefore it is most advisable to harvest rainwater and manage water resources efficiently. It is astounding how these ancient voices speak about modern SDGs such as poverty (SDG 1), zero hunger (SDG 2), clean water (SDG 6), climate action (SDG 13), life on land (SDG 15), peace, justice and strong institutions (SDG 16) in their poems.

Similarly, Valluvar, who is said to have lived between 300 BCE to 5th century CE, and is the author of a celebrated collection of couplets called Thirukkural, reflects on the importance of water: 
வான்நின்றுஉலகம்வழங்கிவருதலால் தான்அமிழ்தம்என்றுணரற்பாற்று

[The world its course maintains through life that rain unfailing gives; thus rain is known as the true ambrosial food of all that lives. It is the bounty of rain, that preserves the world. It is, therefore, worthy of being called ambrosia.]

This poem understands life as immortal, and for that rain is essential, and hence, rain should be called ambrosia or nectar of life (SDG 7). Life on planet earth is manifold. Individual life is mortal but life as a collective process is immortal. This immortality is bestowed by rain. This sophisticated understanding that life depends on rain for its survival, is 'thought-provoking'. From a system's approach, he explains further that rain is essential not only for maintaining life on land but also in the sea (SDG 14).

\section{நெடுங்கடலும்தன்நீர்மைகுன்றும்தடிந்தெழிலி தான்நல்காதாகிவிடின்}

[If clouds restrain their gifts and grant no rain, the treasures fail in the ocean's vast domain. Even the wealth of the vast sea will be diminished if the cloud that has drawn (its waters) up gives them not back again (as rain).]

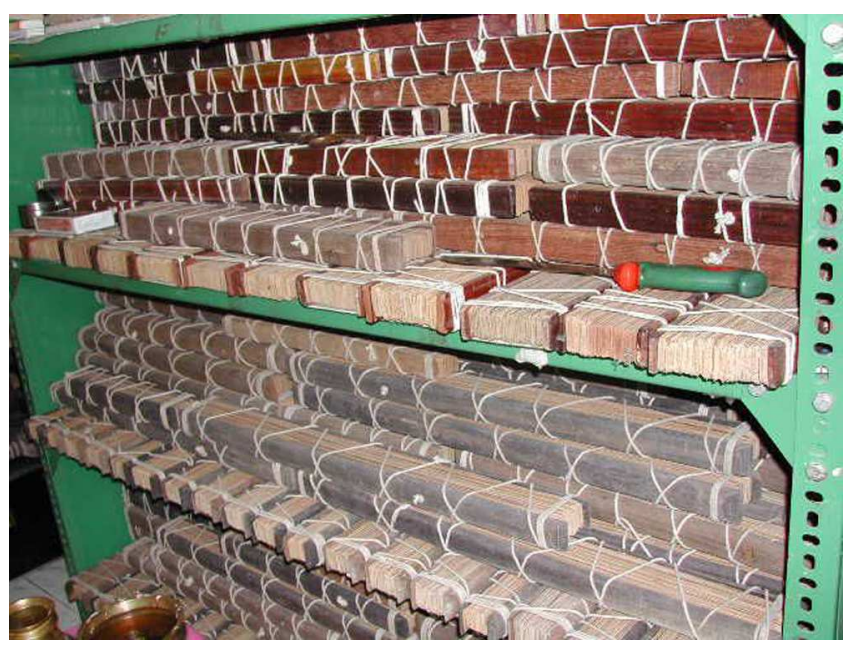

Figure 29.3 Tamil texts in palm leaves.

Source: own photograph. 
This couplet explains that the sea will lose its vitality if rain fails and there is no circulation of water. A detailed understanding of the hydrologic cycle is necessary to compose a poem like this.

\section{நீர்இன்றுஅமையாதுஉலகெனின்யார்யார்க்கும் வான்இன்றுஅமையாதுஒழுக்கு}

[When water fails, functions of nature cease, you say; thus when rain fails, no men can walk in 'duty's ordered way'. If it is said that no one can discharge the duties of life without water, for without rain, there is no life.]

He further elucidates that our world cannot function and civilisation will collapse without rain. Water conservation on land is often discussed yet rarely understood. One may wonder why one should bother when $71 \%$ of the Earth is covered in water, but according to the US Department of Interior's Bureau of Reclamation (2019), ninety-seven per cent of all water on earth is salt water, not suitable for drinking. Only $3 \%$ of water on earth is fresh water, and only $0.5 \%$ of that is available for drinking. The other $2.5 \%$ of fresh water is locked in ice caps, glaciers, the atmosphere, soil, under the earth's surface, or is too polluted for consumption. Further, there is a real fear, a warming planet may lead to 'water wars'. Water is central to all human activities, including food production, no state can allow its water resources to be compromised (Mancosu et al. 2015). Therefore, in a world squeezed dry of water supply, states would go to war to protect their access to water (Levy \& Sidel 2011). At its core, the 'water wars' hypothesis expresses our deepest anxieties about a drought-laden future, wherein desperately thirsty societies take up arms against one another. This is expressed lucidly in the above-mentioned poem of Thiruvaluvar that the order of the society will collapse if water scarcity appears (SDG 16). Knowing the significance of this couplet, Prime Minister of India Mr Narendra Modi quoted this poem in Indian Parliament (Union Budget 2019).

If a civilisation could view water as holy, one has an inkling of the sanctity with which it must have viewed all living beings. Thiruvalluvar (Tirukkural 1886) insists on equal rights for all beings on planet earth in one phrase பிறப்பொக்கும் எல்லா உயிர்க்கும் (all beings are equal) and consequently, speaks out against the indiscriminate killing of life forms through over-fishing, large scale farming and finally biological extinction.

\section{பகுத்துண்டுபல்லுயிர்ஓம்புதல்நுலோர்}

\section{தொகுத்தவற்றுள்எல்லாம்தலை}

[Let those that need to partake your meal guard every-thing that lives; this the chief and sum of lore that hoarded wisdom gives. The chief of all (the virtues), which authors have summed up, is the partaking of food that has been shared with others, and the preservation of the manifold life of other creatures.] 


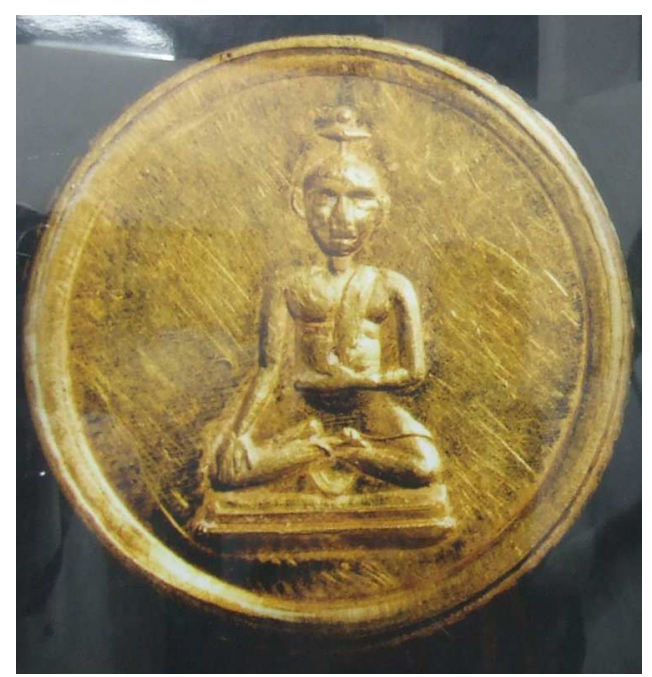

Figure 29.4 An image of Thiruvalluvar in gold coin commissioned by Thomas Scott-Ellis, Collector, British India.

He says that sharing of resources among all beings is considered the best of all virtues (SDG 12).

Probably Thiruvalluvar's voice was the first authentic SDG on hunger (SDG 2). He had the audacity to curse the creator.

\section{இரந்தும்உயிர்வாழ்தல்வேண்டின்பரந்து கெடுகஉலகியற்றியான்}

[If He that shaped the world desires that men should go begging, through life's long course, let him a wanderer be and perish so. If the Creator of the world has decreed even begging as a means of livelihood, may he too go abegging and perish.]

The ancient Tamils treated flora and fauna as equals and were aware of the inter-species connectivity, and its significance. In a poem in the collection Narrinai (2019), a lover hurried to meet his girl under a tree, but she was not to be found there. Questioning her friend on it, the truth comes out that it was a tree that the girl had accidentally planted, then seeing it sprout, had fed and nourished it like a sibling and watched it grow. Now, she feels embarrassed to meet her lover under the watchful eyes of a sibling. The tree that has been mentioned is called Punnai in Tamil (Ballnut in English). This poem in Tamil reads like this: 
விளையாடுஆயமொடுவெண்மணல்அழுத்தி

மறந்தனம்துறந்தகான்முளை

ஆகையநெய்பெய்தீம்பால்பெய்தினிது

வளர்ப்பநும்மினும்சிறந்ததுநுவ்வை

ஆகுமென்றுஅன்னைகூறினள்

புன்னையதுசிறப்பேஅம்ம!!

நாணுதும்நும்மொடுநகையே!

This is just a sampling of ancient Tamil poems and their insistence on sensitivity, sensibility and connectivity to nature and a call for universal brotherhood. This is clearly a nexus perspective emphasising the inter-relatedness and interdependencies of environmental resources and their transitions and fluxes across spatial scales and between compartments.

The ancient Tamil lifestyle was designed in an ecocentric way, and everything, including their gods, are an extension of time and space surrounding them. This concept was called 'Ainthinai' (David 2011) in Tamil (Ecotype/Biotype/ Ecozone). Here the planetary boundaries are clearly defined. For example, ecotypes are characterised by typical flora, fauna, and the tribes living on it. Since an ecosystem essentially defines a way of living, each ecotype has its mood, music and a typical psychological factor called 'deity' or God. This applies to the essential four ecotypes, namely, 'Mullai' (forest ecotype), 'Kurunji (mountain ecozone) 'Marudham' (Agricultural ecozone) and 'Neythal' (Coastal zone). This concept binds all beings in an ecosystem through complex relationships. God, in this concept, is a psychological extension of the ecosystem or a metaphor. This is revealed when the question of a suitable god was sought for a new ecotype called 'Palai or the desert' that appeared several centuries later due to extensive landscaping. It has been explained in literature that Mullai and Kurunji through deforestation led to Palai the desert. The original concept of the four ecotypes was explained in 'Tholkappiam' (David 2011; it has been dated variously between the 3rd century BCE and the 5th century CE). However, a need appeared later to define desert ecosystem with its typical flora, fauna, tribes and a God. This systems innovation approach was certainly a Factor X attempt.

The Tamils had something to say on a circular economy as well. A lady poet called Auvaiyar explains the interconnectivity of ecosystem components clearly in her poem. It reads like this:

வரப்புயர, நீர் உயரும்

நீர் உயர, நெல் உயரும்

நெல் உயர, குடி உயரும்

கு4ி உயர, கோல் உயரும்

கோல் உயர, கோன் உயர்வான். 
Auvaiyar (2019) observes that if the bund of a rice field rises, the water level in the field will rise. When the water level is right for paddy, the plant grows well and yields well. When there is a good harvest people are fed and they are happy. When people are happy, they pay taxes and hence the King becomes powerful. This regenerative approach of Auvaiyar reflects indeed a circular economy, which is in contrast to linear economy, that is a 'take, make, dispose' model.

\section{Key findings}

It is clearly evident that these ancient Tamil texts were forerunners of the modern concept of sustainability, environmental systems innovation, circular economy, nexus approach, an ancient attempt or a model for a society on $\mathrm{X}$ Factor principles.

\section{Recommendation}

By reading those ancient texts, one may wonder how an eco-centric lifestyle has changed so drastically, over the years to a recklessly consuming society, contributing to poverty, environmental degradation and ultimately collapse of ethics and values. Modern India has made great advances through science and technology but simultaneously has been made poorer by losing values which had once sustained it. The objectives of SDGs were to produce a set of universal goals that meet the urgent environmental, political and economic challenges facing our world. Ancient India had all of them. However, not practising them has led to the present sad status. There were debates in India after the end of European colonisation whether India's progress should take the line of decentralisation, sustainability and self-reliance backed by Mohandas Karamchand Gandhi or western style of modernisation backed by Jawarhal Nehru. Gandhi being an ardent believer of ancient wisdom of India based his arguments on universal values that would sustain everyone. His succinct words 'there is enough on Earth for everybody's need, but not enough for everybody's greed' have been proved right. There is no greater truth!

\section{References}

Auvaiyar (2019). [Title in Tamil.] Retrieved from https://ta.wikisource.org/wiki/\% Е0\%AE\%AA\%Е0\%AE\%95\%Е0\%AF\%8D\%E0\%AE\%95\%E0\%AE\%AE\%E0\%AF\%8D:\% E0\%AE\%A4\%Е0\%AE\%AE\%E0\%AE\%BF\%E0\%AE\%B4\%E0\%AF\%8D_\%E0\%AE\%A8\% E0\%AF\%82\%E0\%AE\%B2\%E0\%AF\%8D_\%E0\%AE\%85\%E0\%AE\%B1\%E0\%AE\%BF\% E0\%AE\%AE\%E0\%AF\%81\%E0\%AE\%95\%E0\%AE\%AE\%E0\%AF\%8D.pdf/268 (accessed 12 September 2019).

David, H. (2011). Ainthinai: The Five Regions of the Tamil Country. Traditional Knowledge Systems. Retrieved from https://is.muni.cz/el/1423/podzim2011/HEN612/um/26486599/ TEK-CHAP2.pdf (accessed 12 September 2019). 
Denig, N. (1985). 'On Values' Revisited: A Judeo-Christian Theology of Man and Nature. Landscape Journal, 4(2), 96-105. Retrieved from www.jstor.org/stable/43323106 (accessed 12 September 2019).

El-Haggar, S. M. (2007). Sustainable Industrial Design and Waste Management: Cradle-toCradle for Sustainable Development. Burlington, MA: Elsevier Academic Press.

Encyclopaedia Britannica (2017). Tamil. Retrieved from www.britannica.com/topic/ Tamil (accessed 12 September 2019).

History.com (2019). Maya. Retrieved from www.history.com/topics/ancient-americas/ maya (accessed 12 September 2019).

Jackson, A. (2017). The 10 Most Serious Problems in the World, According to Millennials. Retrieved from www.businessinsider.my/world-problems-most-seriousaccording-to-millennials-2017-8/?r=US\&IR=T\#2-large-scale-conflict-wars-389-9 (accessed 12 September 2019).

Kudapulaviyanar (2019). Purananuru Collections. Retrieved from www.tamilvu.org/ library/11280/html/11280f09.htm (accessed 12 September 2019).

Levy, B. S. \& Sidel, V. W. (2011). Water Rights and Water Fights: Preventing and Resolving Conflicts Before They Boil Over. American Journal of Public Health, 101(5), 778-780.

Lovgren, S. (2019). Angkor Wat's Collapse from Climate Change Has Lessons for Today. Retrieved from www.nationalgeographic.com/news/2017/04/angkor-wat-civilizationcollapsed-floods-drought-climate-change/ (accessed 12 September 2019).

Mancosu, N., Snyder, R. L., Kyriakakis, G. \& Spano, D. (2015). Water Scarcity and Future Challenges for Food Production. Water, 7, 975-992.

Mann, C. C. (2018). 'Silent Spring \& Other Writings' Review: The Right and Wrong of Rachel Carson. The Wall Street Journal, 26 April. Retrieved from www.wsj.com/articles/ silent-spring-other-writings-review-the-right-and-wrong-of-rachel-carson-1524777762 (accessed 12 September 2019).

Narrinai (2019). [Title in Tamil.] Retrieved from www.tamilvu.org/library/11210/html/ 112106bd.htm (accessed 12 September 2019).

O'Connor, L. (2019). Changing Monsoons and Climate: What's the Rain Forecast? Retrieved from https://medium.com/uncclearn/changing-monsoons-and-climate-whatsthe-rain-forecast-8a56c51d6de0 (accessed 12 September 2019).

Pisiranthaiyar (2019). Yanai pukka nilam, Retrieved from http://puram400.blogspot.com/ 2010/09/184.html (accessed 12 September 2019).

SDGs (2019). Sustainable Development Goals. Retrieved from www.undp.org/content/ undp/en/home/sustainable-development-goals.html (accessed 12 September 2019).

Tirukkural (1886). Tirukkural. English translation and commentary by Rev. Dr G.U. Pope, Rev. W. H. Drew, Rev. John Lazarus and Mr F. W. Ellis. London: W. H. Allen \& Co.

US Department of Interior's Bureau of Reclamation (2019). Water Facts - Worldwide Water Supply. Retrieved from www.usbr.gov/mp/arwec/water-facts-ww-water-sup. html (accessed 12 September 2019). 